\title{
Monads for hyperintensionality? A situation semantics for hyperintensional side effects and intra-sentential anaphora
}

\author{
Luke Burke - University of Bamberg and University College London
}

20th April, 2019

\begin{abstract}
P-HYPE, the semantic theory we outline, uses monads from category theory in order to integrate a perspective-sensitive semantic theory with a hyperintensional logic, HYPE (Leitgeb 2018). This enables us to to capture in a fine-grained way the utterer's perspective on other people's perspectives, a phenomenon that (Asudeh and Giorgolo 2016) argue plays a role in natural language. But it also enables us to construct a particular approach to hyperintensionality in natural language within HYPE, since HYPE itself is compatible with different accounts of hyperintensionality in natural language. In P-HYPE, hyperintensionality is modelled as a side effect in the sense of (Charlow 2014) and of consisting in a special type of perspective sensitivity. P-HYPE builds on the account of (Asudeh and Giorgolo 2016), by carving out a notion of perspectives as special sets of situations which can be combined together via a fusion relation. In addition, we briefly illustrate how we can combine P-HYPE with an account of certain kinds of intra-sentential anaphora.
\end{abstract}

Keywords: Attitude verbs, monads, side effects, perspective, hyperintensionality, situation semantics

\section{Introduction: Hyperintensionality as a side effect}

So-called 'hyperintensional' semantic theories allow us to block the free substitution of intensionally equivalent sentences (see (Fox and Lappin 2008) and references therein), such as tautologies and mathematical truths, which, at least in standard frameworks used in natural language semantics (Gallin 1975), express the same function from worlds to truth values. The phenomenon of hyperintensionality might therefore be thought to constitute a 'compositionality challenge' (Zimmermann 2012), in that the naïf semantics employed in standard frameworks cannot compositionally account for the different meaning of distinct mathematical/logical truths and we lack an agreed-upon method of compositionally deriving their meanings. (Shan 2002) had the intuition that we can model many seemingly non-compositional phenomena by using monads, as 'side effects' of computing the main value of an expression. Subsequently, certain linguistic phenomena exhibiting non-determinism, intensionality or state-changing operators have been captured via monads (Charlow 2014). We propose that hyperintensionality be added to this list as a particular kind of side effect, in the sense of (Charlow 2014) and (Shan 2007). (Shan 2007) in particular, includes amongst so-called linguistic side effects certain types of referential opacity and certain expressions whose meaning and compositional contribution is not pre-theoretically transparent. Hyperintensionality is arguably a good example of a linguistic side effect in Shan's sense, since it is not clear what distinction to make between the semantic contribution of logically equivalent statements, how their meanings relate to their truth conditions, and how to characterise their behaviour compositionally. But from both (Charlow 2014) and Shan's list of linguistic side effects, hyperintensionality is conspicuous by its absence. It is high time to fit hyperintensionality into the monadic fold.

Cue the entry of HYPE (Leitgeb 2018), which we discuss in detail later. HYPE is hyperintensional, but does not offer on its own a completely satisfying analysis of hyperintensionality in natural language (as 
(Leitgeb 2018) points out regarding verbs like believe, which he calls 'quasi-syntactic' $\left.{ }^{1}\right)^{2}$ For example, $A \rightarrow(B \rightarrow A)$ is an axiom in HYPE, and so true in all states of every HYPE model. Likewise, $A \rightarrow A$ is an axiom of HYPE. But someone might not believe $A \rightarrow(B \rightarrow A)$, for certain $A, B$. And, again instantiating sentences for $A$ and $B, A \rightarrow(B \rightarrow A)$ and $A \rightarrow A$ might differ in meaning, even though they are logically equivalent in HYPE. ${ }^{3}$ No doubt these problems could be circumvented by a theory of natural language hyperintensionality in HYPE. ${ }^{4}$ That is precisely what we are providing here (however in (Burke 2019) we discuss the particular problem just mentioned), but incorporating perspective sensitivity into HYPE itself. Both P-HYPE and the semantics of (Asudeh and Giorgolo 2016) (from now on $A G$ ), enrich the typed lambda calculus with a reader monad (Shan 2002) defined on the type $p$, of perspective indices. We will discuss how perspective indices are employed later. For now we can just say that to every agent in a discourse there corresponds a perspective index, and that certain terms which are perspective sensitive are interpreted relative to perspective indices. If an expression has type $\alpha$, a perspectivally sensitive expression has type $p \rightarrow \alpha$. The reader monad is a triple $(\diamond, \eta, \star)$. $\diamond: T Y P E \rightarrow T Y P E$, is a type-constructor, which behaves as a special modal operator in Lax logic (Fairtlough and Mendler 1997). ${ }^{5} \diamond$ maps any type $\tau$ to $p \rightarrow \tau$ and, for all $a, b$, maps a function $f: a \rightarrow b$ to a function $\diamond f: \diamond a \rightarrow \diamond b$, such that $(\diamond f)(x)=\lambda i . f(x i){ }^{6} \eta: \tau \rightarrow \diamond \tau$ is a value-constructor that takes a non-monadic value $x: \tau$ and trivially upgrades it to monadic values by forming a constant function from perspective indices to $x$. It is called the unit of the monad:

Definition $1: \eta(x)={ }_{\text {def }} \lambda i . x: p \rightarrow \alpha$

$\star$ ('bind'): $\diamond \tau \rightarrow(\tau \rightarrow \diamond \delta) \rightarrow \diamond \delta$

Finally, $\star$ (called bind) is a polymorphic binary infix operator acting as a sort of functional application:

Definition 2 $: a \star f={ }_{\text {def }} \lambda i . f(a(i))(i) \quad$ where $a: \diamond \tau, f: \tau \rightarrow \diamond \delta$

\subsection{AG's semantic theory}

Consider the sentence (2a), uttered in the scenario (2b)

(2) a. Mary Jane loves Spiderman.

b. Scenario: Mary Jane does not know Peter Parker's secret identity and loves the man she calls 'Peter Parker'. A speaker $\sigma$ who knows or is 'enlightened' (Zimmermann 2005) about Peter Parker's secret identity utters (2a)

According to AG, there is a sense in which (2a) is true, from the perspective of an enlightened utterer, but false from Mary Jane's perspective. (Asudeh and Giorgolo 2016) model this by making certain names perspective relative, so that Mary Jane can associate a distinct denotation with the names 'Spiderman' and 'Peter Parker'. Thus names denote certain people's mental representations-perspectives. We can then imagine a private mental lexicon for each person, consisting of the set of perspectives that a given person associates with terms of her language, which we call that person's perspective. We use 'perspective' ambiguously-both to denote a semantic value in someone's lexicon, and that person's lexicon itself-with the context serving to disambiguate which notion we have

\footnotetext{
1"Once again, the logic and semantics of the system HYPE to be developed in the present article would not be able to contribute to investigations into hyperintensional operators of any such quasi-syntactic kind.”(Leitgeb 2018)

${ }^{2} \mathrm{~A}$ reviewer asks why HYPE is not sufficient to account for hyperintensionality. This paragraph gives some reasons for this conclusion. Another reviewer also writes that HYPE is already hyperintensional, so adding another device (perspectives) for hyperintensionality should be carefully argued for. This paragraph should also be relevant to her question.

${ }^{3}$ For example, 'If I will be late then if I have a coffee then I will be late' has a different meaning from 'If I am late then I am late'. Other examples could be given not involving conditional connectives.

${ }^{4}$ In (Burke 2019) we show that such examples can be dealt with both with HYPE and with P-HYPE. But P-HYPE in addition accounts for a variety of data involving perspective relativity, so is ceterus paribus preferable.

${ }^{5}$ It is an endofunctor, as this is understood in Category theory; that is, a functor that maps a category to itself (in this case the category of types).

${ }^{6}$ Throughout ' $x: \alpha$ ' is read ' $x$ is of type $\alpha$ '
} 


\begin{tabular}{lll}
\hline WORD & DENOTATION & TYPE \\
\hline Mary Jane & $\mathbf{m j}_{\sigma}$ & $e$ \\
Peter Parker & $\mathbf{p p}_{\sigma}$ & $\diamond t \rightarrow e \rightarrow t$ \\
believe & $\lambda c . \lambda s \cdot \mathbf{B}(s, c(\kappa(s)))$ & $\nabla e \rightarrow e \rightarrow t$ \\
love & $\lambda o \cdot \lambda s \cdot \operatorname{love}(s, o(\kappa(s)))$ & $\nabla e$ \\
Spider-Man & $\lambda i .\left\{\begin{array}{l}\mathbf{s m}(\mathbf{i}) \quad \text { if } i=\kappa(m j) \\
\mathbf{p p}(\mathbf{i}) \quad \text { if } i=\kappa(\sigma)\end{array}\right.$
\end{tabular}

Table 1 Lexicon of $\sigma$, the enlightened speaker

in mind. Consider the lexicon (Table 1) of the enlightened speaker $\sigma$ of (2a). Plain names are subscripted with $\sigma$ to indicate that this is the denotation of that name for $\sigma$. The names which are type $\checkmark e$ have different denotations, depending on the perspective index they are fed. AG suppose that certain names vary in perspective but others do not. Those which do not vary in perspective have something like a default status, in the following sense: if someone becomes enlightened, and learns, for example that Spiderman and Peter Parker are one and the same thing, then they will, by and large, just use plain 'Peter Parker', and this name will thence have default status, with 'Spiderman'. ${ }^{7}$ Notice the $\kappa$ operator in the denotation of believe and love. $\kappa$ has the following interpretation (where $D_{e}$ is the domain of individuals of a model):

$$
\forall x \in D_{e}(\kappa(x) \in P)
$$

Perspective sensitive expressions that scope below $\kappa$ are interpreted relative to the perspective index corresponding to the subject of the attitude report (see Table 1). Expressions that scope above $\star$, are interpreted relative to the default perspective of the utterer. Let us consider the two readings of (2a). The false reading of (2a) is represented by (4), which $\beta$-reduces to (5), and the true reading is represented by (6), which $\beta$-reduces to (7):

$$
\begin{aligned}
& \text { love }\left(m j_{\sigma}, \lambda i .\left\{\begin{array}{ll}
\mathbf{s m}(\mathbf{i}) & \text { if } i=\kappa(m j) \\
\mathbf{p p}(\mathbf{i}) & \text { if } i=\kappa(\sigma)
\end{array}(\kappa(m j))\right)\right. \\
& \text { love }\left(m j_{\sigma}, \operatorname{sm}(\kappa(m j))\right) \\
& \left(\lambda i .\left\{\begin{array}{ll}
\mathbf{s m}(\mathbf{i}) & \text { if } i=\kappa(m j): P \\
\mathbf{p p}(\mathbf{i}) & \text { if } i=\kappa(\sigma): P
\end{array} \star \lambda z . \eta(\operatorname{love}(m j, z))\right)\right)(\kappa(\sigma))
\end{aligned}
$$

\footnotetext{
${ }^{7}$ A reviewer suggests that giving "perspective sensitive" proper names a different type from ordinary proper names could be seen as an undesirable complication. We could in fact give all proper names uniformly type $\diamond e$. However, the distinction in type is motivated by the distinction in function between certain uses of proper names in given contexts. The type assignment is relative to a given context, because, as (Asudeh and Giorgolo 2016) point out, whether a name seems to have a double life, like Peter Parker, depends on what we assume to be true about the name and its bearer. The type assignment therefore simply reflects this reality (that certain names are 'controversial' and others are not). The reviewer also asks whether P-HYPE has something to say about misunderstanding that can arise in dialogue where dialogue participants understand different referents for a particular utterance of a proper name. This usually happens when not enough information is salient in the conversational context to determine which Mary, or John, etc, is meant. As such, it is more a question about the pragmatic discourse context than about the semantic competence that a name user has, and so we haven't analysed this phenomenon. In P-HYPE each Mary would be associated with a distinct lexical entry, but some Mary's might be associated with two distinct lexical entries, if someone thinks that there are two people corresponding to one Mary. (Asudeh and Giorgolo 2016) discuss such cases and offer a suggested solution.
} 
Since Spiderman in (6) scopes above $\star$ and above $\kappa$, it is interpreted relative to the default perspective index, which is the index of the speaker, who they assume in their model to be enlightened. They thus stipulate that the speaker's perspective is the one fed to an expression of the form $a \star f$, which by definition denotes $\lambda i . f(a(i))(i)$, and thus, if the speaker's index is $j$, we always evaluate some expression of the form $a \star f$ at $j .{ }^{8}$ When, however, a perspective relative expression scopes below the function $f$ in $a \star f$, it is caught by the $\kappa$ operator.

\section{P-HYPE: a combination of HYPE and perspective sensitivity}

HYPE (Leitgeb 2018) is a logic which employs states/situations. ${ }^{9}$ States may be like classical possible worlds, but may also be partial (or gappy)-verifying neither a formula nor its negation- and inconsistent (or glutty)- verifying a formula and its negation. One nice feature of HYPE is that it behaves entirely classically at a subset of states; as such, linguistic analyses couched in classical logics can be transferred to HYPE. HYPE incorporates special incompatibility $\perp$ and fusion operators $\circ$ in the satisfaction clauses for negation and the conditional, somewhat like (Veltman 1985: pp. 202-7):

1. $\circ: S \times S \rightarrow S$ is a partial commutative, associative binary function (called fusion), such that:

- Either $s \circ s^{\prime}$ is undefined, or $s \circ s^{\prime}$ is defined (and hence in $S$ ) in which case it is required that $V\left(s \circ s^{\prime}\right) \supseteq V(s) \cup V\left(s^{\prime}\right)$.

$-s \circ s$ is defined, and $s \circ s=s$.

2. $\perp$ is a binary symmetric relation on $S$ (the incompatibility relation), such that:

- If there is a $v$ with $v \in V(s)$ and $\bar{v} \in V\left(s^{\prime}\right)$, then $s \perp s^{\prime}$.

- If $s \perp s^{\prime}$ and both $s \circ s^{\prime \prime}$ and $s^{\prime} \circ s^{\prime \prime \prime}$ are defined, then $s \circ s^{\prime \prime} \perp s^{\prime} \circ s^{\prime \prime \prime}$.

$\circ$ gives rise to a partial order $\leq$, such that, for all $s, s^{\prime} \in S, s \leq s^{\prime}$ iff $s \circ s^{\prime}$ is defined and $s \circ s^{\prime}=s^{\prime}$. Importantly, truth is monotonic under fusion extension: for all $s$, if $s \models A$ and $s \circ s^{\prime}$ is defined, then $s \circ s^{\prime} \models A$.

Variable assignments $\rho$ and their modified variants $\rho(d / x)$ behave as in Classical Predicate logic. Satisfaction of a formula $\phi$ is defined relative to a state and a variable assignment (written: $s, \rho=\phi$ ), and the clauses for the logical symbols are as usual, except for $\neg$ and $\supset$, which have a distinctly modal flavour: $s, \rho=\neg A$ iff for all $s^{\prime}:$ if $s^{\prime}, \rho=A$ then $s \perp s^{\prime}$ and $s, \rho \models A \supset B$ iff for all $s^{\prime}:$ if $s^{\prime}, \rho \models A$ and $s \circ s^{\prime}$ is defined, then $s \circ s^{\prime} \models B$. The reader should consult (Leitgeb 2018) for more details, including the relationship of HYPE to Classical, Intuitionistic and four-valued logic.

\subsection{Introducing P-HYPE: a combinination of HYPE and AG's perspective- sensitive semantics}

In P-HYPE, we require the usual hierarchy of typed domains familiar from (Gallin 1975), whose elements correspond to different kinds of entities. To this end, let $T Y P E$ be the smallest set such that:

\footnotetext{
${ }^{8}$ The technical stipulation they make is grounded in certain claims about perspective relativity, such as the claim that sentences or expressions which are perspective relative are usually relative to the perspective of the utterer of them, and if they are relative to other perspectives, they are relative either to individuals salient in some group within a given context, or are relative to the perspective of the subject of the sentence. We won't assess these claims here, but suffice to say that they have been discussed and broadly endorsed by researchers working on perspective relativity.

${ }^{9}$ We will use the words 'states' and 'situations' interchangeably.
} 
3. $\alpha \in T Y P E$ implies $\diamond \alpha \in T Y P E$

For each type, there is a countable set of constants, and variables, and the set of constants (variables) is the union of the sets of constants (variables) of each type:
- $C O N=\bigcup_{\tau \in T Y P E} C O N_{\tau}$
- $V A R=\bigcup_{\tau \in T Y P E} V A R_{\tau}$

The set of terms is the union of the sets of terms of type $\tau$, for arbitrary $\tau$ :

$T E R M=\bigcup_{\tau \in T Y P E} T E R M_{\tau}$

The set $T E R M_{\tau}$ for arbitrary type $\tau$ is then defined as follows:

- $c \in C O N_{\tau}$ implies $c \in T E R M_{\tau}$;

- $x \in V A R_{\tau}$ implies $x \in T E R M_{\tau}$;

- $\tau=\alpha \rightarrow \beta, t \in T E R M_{\beta}$ and $x \in V A R_{\alpha}$ imply $(\lambda x . t)_{\tau} \in T E R M_{\tau}$

- $t \in T E R M_{\alpha \rightarrow \tau}$ and $u \in T E R M_{\alpha}$ implies $(t u) \in T E R M_{\tau}$.

- $A \in T E R M_{t}$ and $x \in V A R_{\alpha}$ implies

- $A \in T E R M_{t}$ implies $\neg_{c} A \in T E R M_{t}$ $(\forall x . A) \in T E R M_{t}$

- $A, B \in T E R M_{t}$ implies $(A * B) \in T E R M_{t}$, for $* \in\{\wedge, \vee, \rightarrow\}$

- $x \in T E R M_{e}$ implies $D O X_{x} \in T E R M_{s \rightarrow s \rightarrow t}$ and $P R O V_{x} \in T E R M_{s \rightarrow s \rightarrow t}$

- $\circ \in T E R M_{s \rightarrow s \rightarrow s}$

- $\leq \in T E R M_{s \rightarrow s \rightarrow t}$

- $\pi \in T E R M_{p \rightarrow s \rightarrow s \rightarrow t}$

- $\kappa \in T E R M_{e \rightarrow s \rightarrow p}$

- $\perp \in T E R M_{s \rightarrow s \rightarrow t}$

Suppose we have sets $S, D, P$, where:

- $S=S_{c} \uplus S_{n} \neq \emptyset$, where $S_{n}$ is the set of glutty or gappy states ('non-classical' states) and $S_{c}$ is the set of classical states.

- $D \neq \emptyset$ is the domain of individuals $\quad-P \neq \emptyset$ is the set of perspective indices.

Consider functions $\kappa, \pi$ :

- $\kappa: D \rightarrow S \rightarrow P$, is a function that associates a unique perspective index $\kappa(d)(s)$ to each individual $d$ in a state $s$. (refinements possible)

- $\pi: P \rightarrow S \rightarrow \mathscr{P}(S)$ maps every perspective index $\kappa(d)(s) \in P$ and states $s \in S$ to a set of states $\pi(\kappa(d)(s))(s) \subseteq S$, the perspective set or $p$-set of $d$ at $s$. We present $\pi$ in the term language as a characteristic function of type $P \rightarrow S \rightarrow S \rightarrow\{1,0\}$.

We also have a distinguished perspective index:

- $E \in P$ is called enlightened and is such that for all $s, s^{\prime}: \pi(E)(s)\left(s^{\prime}\right)$ iff $s^{\prime} \in\{s\}$, whence $s^{\prime}=s$

The (optional) 'privacy' condition of $A G$ :

- For all $d, d^{\prime} \in D$ for which $d \neq d^{\prime}$, and all $s \in S, \pi(\kappa(d, s), s) \cap \pi\left(\kappa\left(d^{\prime}, s\right), s\right)=\emptyset$.

HYPE negation involves $\perp$ and the conditional involves $\circ$, and can be defined directly in our type-theoretic framework, in the object language: 


\begin{tabular}{|c|c|c|}
\hline WORD & DENOTATION & ABBREVIATION \\
\hline Spiderman & $\lambda i, s .\left\{\begin{array}{ll}s m(i)(s) & \text { if } \exists s \in S . i=\kappa(h, s) \\
p p(i)(s) & \text { else } \exists s \in S . i=E\end{array}: \diamond e\right.$ & $s m$ \\
\hline Inductive & $\lambda x, \lambda j, s .\left\{\begin{array}{ll}I(j)(s)(x(j)) & \text { if } \exists s \in S \cdot j=\kappa(h)(s) \\
\text { finite }(j)(s)(x(j)) & \text { else } \exists s: S \cdot j=E\end{array} \quad: \diamond e \rightarrow \diamond(s \rightarrow t)\right.$ & inductive \\
\hline Love & $\lambda y, x, i, s . \quad \forall s^{\prime}\left[\pi(i)(s)\left(s^{\prime}\right) \rightarrow\right.$ love $\left.\left.(i)\left(s^{\prime}\right)(x)(y)\left(\kappa\left(x, s^{\prime}\right)\right)\right)\right]: \diamond e \rightarrow e \rightarrow \diamond(s \rightarrow t)$ & love \\
\hline Believe/Think & $\begin{array}{l}\lambda p, x, i, s . \forall s^{\prime}\left[s \leq s^{\prime} \wedge \pi(i)(s)\left(s^{\prime}\right) \rightarrow\right. \\
\left.\forall s^{\prime \prime}\left[D O X_{x} s^{\prime} s^{\prime \prime} \rightarrow p\left(\kappa(x)\left(s^{\prime \prime}\right)\right)\left(s^{\prime \prime}\right)\right]\right]: \diamond(s \rightarrow t) \rightarrow e \rightarrow \diamond(s \rightarrow t)\end{array}$ & think \\
\hline Prove & $\begin{array}{l}\lambda p, x, i, s . \forall s^{\prime}\left[s \leq s^{\prime} \wedge \pi(i)(s)\left(s^{\prime}\right) \rightarrow\right. \\
\left.\forall s^{\prime \prime}\left[P R O V_{x} s^{\prime} s^{\prime \prime} \rightarrow p\left(\kappa(x)\left(s^{\prime \prime}\right)\right)\left(s^{\prime \prime}\right)\right]\right]: \diamond(s \rightarrow t) \rightarrow e \rightarrow \diamond(s \rightarrow t)\end{array}$ & $\lambda p, x, i . p r o v e\left(i, x, p\left(\kappa\left(x, s^{\prime \prime}\right)\right)\right.$ \\
\hline the primes & $\lambda i, s . \iota x:$ prime.number $(i)(s)(x(i)): \diamond e$ & the.primes \\
\hline
\end{tabular}

Table 2 Simplified lexical entries

$$
\text { - }\left(\neg A_{s \rightarrow t}\right)=\lambda s . \forall s^{\prime}\left(\left(A_{s \rightarrow t}\right)\left(s^{\prime}\right) \rightarrow s \perp s^{\prime}\right) \quad \text { - }\left(A_{s \rightarrow t} \supset B_{s \rightarrow t}\right)=\lambda s . \forall s^{\prime}(s \leq
$$

A frame $\mathcal{F}=\langle S, \mathscr{D}, \kappa, P, E, \pi, \circ, \perp\rangle$ based on $D, S$ and $P$ is a family of sets $\mathscr{D}=\left\{D_{\alpha} \mid \alpha \in T Y P E\right\}$ such that $D_{e}=D, D_{t}=\{1,0\}, D_{s}=S$ and $D_{p}=P$, and $D_{\alpha \rightarrow \beta} \subseteq\left\{f \mid f: D_{\alpha} \rightarrow D_{\beta}\right\}$ for each type $\alpha \rightarrow \beta$ and $E, \kappa, \pi, \circ, \perp$ are as above. A $P$-HYPE model is a structure $\mathfrak{M}=\langle\mathcal{F}, m\rangle$ where $\mathcal{F}$ is as above and $m: \Pi \alpha . C O N_{\alpha} \rightarrow D_{\alpha}$ is an interpretation of constants. An assignment over $\mathfrak{M}$ is a function $\rho: \Pi \alpha . V A R_{\alpha} \rightarrow D_{\alpha}$. Variable assignments $\rho$ and their modified variants $\rho(d / x)$ (i.e $x \mapsto d$ ) behave as in Classical Predicate logic. There exists an admissible valuation $V$, which assigns to each assignment $\rho$ over $\mathfrak{M}$ and each term $A_{\alpha}$ a value $V_{a}\left(A_{\alpha}\right) \in D_{\alpha}$, provided the following conditions are met:

- $V_{\rho}\left(x_{\alpha}\right)=\rho\left(x_{\alpha}\right)$

- $V_{\rho}\left(\forall x_{\alpha} A\right)=\bigcap_{d \in D_{\alpha}} V_{\rho(d / x)}(A)$

- $V_{\rho}\left(A_{\alpha \rightarrow \beta} B_{\alpha}\right)=V_{\rho}\left(A_{\alpha \rightarrow \beta}\right) V_{\rho}\left(B_{\alpha}\right)$
- $V_{\rho}\left(c_{\alpha}\right)=m\left(c_{\alpha}\right)$

- $V_{\rho}\left(\exists x_{\alpha} A\right)=\bigcup_{d \in D_{\alpha}} V_{\rho(d / x)}(A)$

- $V_{\rho}\left(A_{\alpha} \equiv B_{\alpha}\right)=\left\{s \mid V_{\rho}\left(A_{\alpha}\right)=V_{\rho}\left(B_{\alpha}\right)\right\}$

- $V_{\rho}\left(\lambda x_{\alpha} A_{\beta}\right)=$ the function $f$ on $D_{\alpha}$ whose value at $d \in D_{\alpha}$ is equal to $V_{\rho^{\prime}} A_{\beta}$, where $\rho^{\prime}=\rho(d / x)$

\subsection{Lexical entries and example}

There are three comments to make about these lexical entries in Table 2. Firstly, $h$ denotes 'Harold' (who features in our examples), ' $E$ ' denotes the enlightened perspective and ' $u$ ' denotes the perspective index of the utterer of a sentence. The enlightened perspective index is the perspective index which, if supplied to an expression whose denotation takes a perspective index as an argument, returns the intension of that expression. Secondly, many of these lexical entries are simplified. For example, we are assuming (for expository simplicity) that prove is a guarded universal quantifier over worlds-though we haven't specified what sort of universal quantifier it is-and that Prove is factive, and so presupposes the 
truth of its complement. Thirdly, a crucial aspect of the lexical entries for verbs, is that we are able to formalise the intuition of $\mathrm{AG}$ that such complements are always interpreted relative to a perspective which the utterer thinks is the perspective of another person. Consider the denotation of believe, which combines with a proposition of type $\diamond(s \rightarrow t)$ (i.e, a function from perspective indices to states to truth values), an individual and a perspective index. We assume that, in the case of propositional attitude verbs, this perspective index must always be the utterer's perspective index. Where $u$ is the utterer's perspective index at state $s$, Believe then universally quantifies over both (i) all the states $s^{\prime} \geq s$, such that $s^{\prime} \in \pi(u)(s)$, where $s$ is the world in which the sentence is being evaluated and (ii) all the states $s^{\prime \prime}$ which are doxastically accessible from $s \circ s^{\prime}$. x believes $p$ is then true iff $p$ is true in $s^{\prime \prime}$ relative to the perspective index associated with $x$ at $s^{\prime \prime}$.

Consider (8) and (9): ${ }^{10}$

Harold proves that the primes are not Harold proves that the primes are infinite.

Using the lexical entries above, we can derive (10) for a sentence like (8) and (11) for a sentence like (9):

$$
\begin{aligned}
& \lambda s . \forall s^{\prime}\left[s \leq s^{\prime} \wedge s^{\prime} \in \pi(u, s)\right] \rightarrow \forall s^{\prime \prime}\left[P R O V_{x} s^{\prime} s^{\prime \prime} \rightarrow\right. \\
& \left.\neg I\left(\kappa\left(h, s^{\prime \prime}\right)\right)\left(s^{\prime \prime}\right)\left(\iota x . p r i m e . n u m b e r\left(x\left(\kappa\left(h, s^{\prime \prime}\right)\right)\right)\right)\right] \\
& \lambda s . \forall s^{\prime}\left[s \leq s^{\prime} \wedge s^{\prime} \in \pi(u, s)\right] \rightarrow \forall s^{\prime \prime}\left[P R O V_{x} s^{\prime} s^{\prime \prime} \rightarrow\right. \\
& \left.\neg \text { finite }\left(\kappa\left(h, s^{\prime \prime}\right)\right)\left(s^{\prime \prime}\right)\left(\iota x . p r i m e . n u m b e r\left(x\left(\kappa\left(h, s^{\prime \prime}\right)\right)\right)\right)\right]
\end{aligned}
$$

Crucially, (10) and (11) will differ in truth value, if Harold associates distinct denotations with inductive and finite.

If we scope both inductive and the primes above $\star$, we derive ((12)), the interpretation on which both are not inductive, and the prime numbers are interpreted relative to $E$ and within the $p$-set of associated with $E$ :

$$
\left(\eta\left(\lambda x, \lambda j, s .\left\{\begin{array}{ll}
I(j)(s)(x(j)) & \text { if } \exists s \in S \cdot j=\kappa(h)(s) \\
\text { finite }(j)(s)(x(j)) & \text { else } \exists s: S \cdot j=E
\end{array}\right)\right) \star\right.
$$

$\lambda R . \lambda j, s . \begin{cases}\iota x: \text { prime.number }(j)(s)(x(j)) & \text { if } \exists s \in S . j=\kappa(h)(s) \\ \iota x: \text { prime.number }(j)(s)(x(j)) & \text { else } \exists s: S . j=E\end{cases}$

$\left.\lambda y . \operatorname{not}\left(\lambda i, s . \forall s^{\prime}, s^{\prime \prime}\left(s \leq s^{\prime} \wedge \pi(i)(s)\left(s^{\prime}\right) \wedge\left(P R O V_{x} s^{\prime} s^{\prime \prime}\right) \rightarrow R(\eta(y))\left(s^{\prime \prime}\right)\left(\kappa(h)\left(s^{\prime \prime}\right)\right)\right)\right]\right)$

Feeding $E$ to (12) reduces to (13) :

$$
\lambda s . \forall s^{\prime \prime}\left(P R O V_{x} s s^{\prime \prime} \rightarrow \neg_{c} \text { finite }(E)\left(s^{\prime \prime}\right)\left(\iota x . p r i m e . n u m b e r(E)\left(s^{\prime \prime}\right)(x(E))\right)\right]
$$

The interpretation on which both are not inductive, and the prime numbers are interpreted relative to $u$ and within the $p$-set of associated with $u$ is as follows:

$$
\begin{aligned}
& \lambda s . \forall s^{\prime}\left[s \leq s^{\prime} \wedge s^{\prime} \in \pi(u)(s)\right] \rightarrow \forall s^{\prime \prime}\left[P R O V_{x} s^{\prime} s^{\prime \prime} \rightarrow\right. \\
& \left.\neg I(u)\left(s^{\prime \prime}\right)\left(\iota x . p r i m e . n u m b e r(u)\left(s^{\prime \prime}\right)(x(u))\right)\right]
\end{aligned}
$$

There remain two 'mixed' interpretations:

$$
\begin{aligned}
& \lambda s . \forall s^{\prime}\left[s \leq s^{\prime} \wedge s^{\prime} \in \pi(u)(s)\right] \rightarrow \forall s^{\prime \prime}\left[P R O V_{x} s^{\prime} s^{\prime \prime} \rightarrow\right. \\
& \left.\neg I(u)\left(s^{\prime \prime}\right)\left(\iota x . p r i m e . n u m b e r\left(\kappa(h)\left(s^{\prime \prime}\right)\right)\left(s^{\prime \prime}\right)\left(x\left(\kappa(h)\left(s^{\prime \prime}\right)\right)\right)\right)\right]
\end{aligned}
$$

\footnotetext{
${ }^{10}$ In (Cresswell 1985:p.82) a set is defined to be 'finite' iff it cannot be put into a one-one correspondence with a proper subset of itself, and a set is 'inductive' iff it can be put into a one-one correspondence with a proper initial segment of the natural numbers. The inductive sets and the finite sets are provably equivalent in ZFC.
} 


$$
\begin{aligned}
& \lambda s . \forall s^{\prime}\left[s \leq s^{\prime} \wedge s^{\prime} \in \pi(u)(s)\right] \rightarrow \forall s^{\prime \prime}\left[P R O V_{x} s^{\prime} s^{\prime \prime} \rightarrow\right. \\
& \left.\neg I\left(\kappa(h)\left(s^{\prime \prime}\right)\right)\left(s^{\prime \prime}\right)\left(\iota x . p r i m e . n u m b e r(u)\left(s^{\prime \prime}\right)(x(u))\right)\right]
\end{aligned}
$$

Having introduced P-HYPE, we would like to respond to a few remarks made by reviewers. A reviewer asks how well the view of perspectives outlined deals with cases where on one perspective a proper name does not denote at all whereas on other perspectives it does. In P-HYPE any user of a name would, if they can use the name, associate a perspective with the name, even if the user considers the name to be non-denoting. Thus the difference between the two cases depends on whether someone has a metalinguistic belief to the effect that, for example, 'Zeus' denotes. The reviewer asks also how in P-HYPE we can account for someone, let's call her Mary, who considers Jupiter to be identical to Zeus but does not believe that there is such a god. There are various ways we might consider accounting for this, however, we have not decided which is best, and so suggest just one possibility. Suppose that a perspective index can be supplied for certain fictions which are salient in a discourse context, or perhaps certain people's views of those fictions. Then the identity of Zeus and Jupiter would be true relative to the perspective index associated with this fiction in the mind of Mary, and the perspective index supplied for her belief that there are no gods would be her very own dedicated perspective index. This supports the intuition that when we speak truth about fiction, we often do so from within a particular fiction, or from some messy combination of the fiction with other beliefs we have (Lewis 1978).

Another reviewer had trouble seeing what the value of HYPE is in the semantics outlined, and asks how perspectives are acquired and modified. These are good questions, which I attempt to answer in (Burke 2019). Suffice to say that the role of the fusion operator in HYPE actually forms a crucial role in my account, in that it allows us to combine and enlarge perspectives. Thus the HYPE framework is very useful for this. Furthermore, as discussed earlier, the HYPE framework is compatible with the sorts of analyses that semanticists have given of intensional phenomena (for example in (Heim \& von Fintel 2011)), which usually employ classical logic.

Finally, the same reviewer questions a perceived 'relativism' that she thinks our semantics entails, by having both the reference of names and predicates as being dependent on a perspective. Here we would like to clarify and expand upon this objection. According to the objection, if we allow predicates to be perspective relative across the board, we predict that sentences like (17) could be true relative to some perspectives, and therefore not plainly false:

There are ten natural numbers.

But this objection misunderstands what we intend by saying something is true relative to a perspective. To say this is simply to say that there exists a speaker whose whose mental model is constituted in such a way as to associate certain representations with certain sentence parts. ${ }^{11}$ This is compatible with (17) being plainly false, and the truth/falsity simpliciter of a sentence is determined relative to the enlightened perspective index. We are not denying, unlike MacFarlane (2014), that certain sentences are true simpliciter. For us, (17) is strictly speaking false, however it is true (or coherent) relative to some bizarre perspective index, which is unlikely to be associated with all but the strangest of individuals, and unlikely to be salient in any context. In fact, in P-HYPE we have $E$, the dedicated, designated perspective index associated with the enlightened perspective, which allows us to reason about 'plain truth' by simply providing the ordinary intension associated with a sentence constituent. So the charge of relativism is not correct.

\subsection{Anaphora in P-HYPE: the State.Set monad}

In this final section we will take a quick look at how intra-sentential anaphora can be analysed in P-HYPE.

Consider (18), on the reading in which he is anaphoric to a man she thinks is Peter Parker:

\footnotetext{
${ }^{11}$ We could even talk of the coherence of a statement relative to a perspective, instead of the truth of a statement relative to a perspective, if this is less liable to confuse.
} 
Mary Jane loves a man she believes is Peter Parker. She believes he isn't Spiderman.

In the standard intensional semantics, at least in which, following (Kripke 1980), co-referring names are given the same semantic value and identity statements are necessarily true or necessarily false, the second sentence of ((18)) denotes the empty set. Clearly, however, there is a sense in which Mary Jane can believe that Spiderman is not Peter Parker. (Lewis 1979) uses centred worlds to account for cases like this, in which someone does not believe a true identity statement and these can potentially be combined with the dynamic semantics of (Groenendijk and Stokhof 1991) in order to model sentences such as (18). Our semantics can also be enriched with a monad that has been used to model anaphora (Charlow 2014): the State Set monad, which is defined as follows:
1. $\diamond \alpha=$
$R \rightarrow(\alpha \times R) \rightarrow t$
2. $\eta(a)=\lambda r .\{\langle a, r\rangle\}$
3. $m \star \lambda v \cdot \pi=$
$\lambda s . \bigcup_{\left\langle a, s^{\prime}\right\rangle \in m s} \pi[a / v] s^{\prime}$

Here, following (Charlow 2014), $R$ is the set of stacks, which are linear sequences of discourse referents (drefs), and $r, r^{\prime}$ are variables over stacks. The stack $r$ can be extended with $x$ (written $r \widehat{x}$ ). The last member of $r$ is the most recently introduced dref, and is notated $r_{\top}$, such that $(r \widehat{a})_{\top}=a$. Following (Charlow 2014), we adopt the definitions in (19) (where pro is the denotation of a pronoun, which picks out the most recent dref), which allow for indefinites to bind pronouns intra-sententially:

$$
\begin{array}{ll}
\text { a. } & \text { a.man }=\lambda r . \bigcup\{\langle x, r\rangle \mid \operatorname{man}(x)\}) \\
\text { b. } & m^{\triangleright}=m \star \lambda v, r . \eta(v) \widehat{r v}=m \star \lambda v, r .\{\langle v, \widehat{r v}\rangle\} . \\
\text { c. } & \text { pro }:=\lambda r .\left\{\left\langle r_{\top}, r\right\rangle\right\}
\end{array}
$$

We can in fact derive (20) from these definitions (see (Charlow 2014:p.47)), which enables a $\triangleright$-shifted $m$ to bind a pronoun in its scope:

$$
m^{\triangleright} \star \lambda v . p r o \star \lambda u . \pi=m^{\triangleright} \star \lambda v \cdot \pi[v / u]
$$

With these definitions in place (and the fact, LeftId, that in any monad $\eta(a) \star f=f a$ ), we can give the

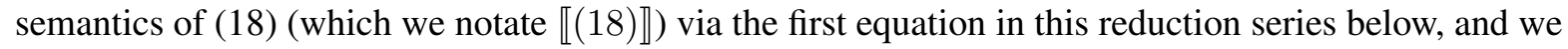
can compositionally derive this lambda term from the parts of (18) (though space reasons precludes us doing so here: ${ }^{12}$

$$
\begin{aligned}
& \llbracket(18) \rrbracket=(\eta(m j))^{\triangleright} \star \lambda x \text {.pro } \star \lambda x^{\prime} \text {.pro } \star \lambda x^{\prime \prime} . \text { a.man }{ }^{\triangleright} \star \lambda y . p r o \star \lambda y^{\prime} . s m \star \lambda z . p r o \star \lambda y^{\prime \prime} . \\
& \eta\left[\left(\operatorname{love}(x, y) \wedge \operatorname{think}\left(x^{\prime}, y^{\prime}=z\right)\right), \wedge \operatorname{think}\left(x^{\prime}, y^{\prime \prime} \neq \operatorname{sm}\left(\kappa\left(x^{\prime}\right)\left(s^{\prime \prime}\right)\right)\right]\right. \\
& ={ }_{\text {by }(20)}(\eta(m j))^{\triangleright} \star \lambda x . a . m a n^{\triangleright} \star \lambda y \cdot \eta(s m) \star \lambda z . p r o \star \lambda y^{\prime \prime} . \\
& \eta\left[(\operatorname{love}(x, y) \wedge \operatorname{think}(x, y=z)), \wedge \operatorname{think}\left(x, y^{\prime \prime} \neq \operatorname{sm}\left(\kappa(x)\left(s^{\prime \prime}\right)\right)\right]\right. \\
& ={ }_{\text {by LeftId }} \text { a.man }{ }^{\triangleright} \star \lambda y \cdot \text { pro } \star \lambda y^{\prime \prime} \text {. } \\
& \lambda r .\left\{\left\langle\left[(\operatorname{love}(m j, y) \wedge \operatorname{think}(m j, y=p p)), \wedge \operatorname{think}\left(m, y^{\prime \prime} \neq \operatorname{sm}\left(\kappa(m)\left(s^{\prime \prime}\right)\right)\right], \widehat{r} m \widehat{\jmath} y\right\rangle\right\}\right. \\
& ={ }_{b y(20)} a \cdot m^{\triangleright} n^{\triangleright} \star \lambda y, r \text {. } \\
& \left\{\left\langle\left[(\operatorname{love}(m j, y) \wedge \operatorname{think}(m j, y=p p)), \wedge \operatorname{think}\left(m j, y \neq s m\left(\kappa(m)\left(s^{\prime \prime}\right)\right)\right] \widehat{r} m \widehat{\jmath y}\right\rangle\right\}\right.
\end{aligned}
$$

The last member of this sequence, reduces by definition of $\star$ to (21), which consists of a function from stacks to sets of pairs consisting of booleans and stacks updated with the drefs $m j$ and $a$, if $a$ is a man:

$$
\begin{aligned}
& \lambda r . \bigcup_{\left\langle a, r^{\prime}\right\rangle \in\left(a . m a n^{\triangleright}(r)\right)}\{\langle[(\operatorname{love}(m, y) \wedge \operatorname{think}(m, a=p p)), \wedge \operatorname{think}(m, a= \\
& \left.\left.\left.\operatorname{sm}\left(\kappa(m)\left(s^{\prime \prime}\right)\right)\right] \hat{r m} \hat{\jmath} a\right\rangle\right\}
\end{aligned}
$$

\footnotetext{
${ }^{12} \mathrm{~A}$ compositional derivation of this lambda term can be given via higher order continuations, much along the lines that (Barker and Shan 2014) use these to account for multiple pronouns.
} 


\section{Conclusion}

We have presented P-HYPE and discussed a monadic approach to hyperintensionality. Elsewhere (Burke 2019) we have discussed the role of perspectives in our account, and phenomena such as metalinguistic focus negation. Here we in addition extend our fragment to cover intra-sentential anaphora, and we have responded to some questions that arise in our account of hyperintensionality. In future work we hope to explore the P-HYPE account of hyperintensionality by comparing it with alternative frameworks which are similar in some respects, such as that of truthmaker semantics (Fine 2017).

\section{References}

Asudeh, A. and G. Giorgolo (2016). Perspectives. Semantics \& Pragmatics 9(21), 1-53.

Barker, C. and C.-c. Shan (2014). Continuations and natural language, Volume 53. Oxford studies in theoretical linguistics, OUP.

Burke, L. (2019). On P-HYPE: a compositional extension of hype. Analysing hyperintensionality and metalinguistic focus via multiple side effects. Studia Logica. Trends in Logic XVIII. Logic in High Definition: Developing Fine-Grained Semantics.

Charlow, S. (2014). On the semantics of exceptional scope. PhD diss,. New York University.

Cresswell, M. J. (1985). Structured meanings. Cambridge, MA: MIT.

Fairtlough, M. and M. Mendler (1997). Propositional Lax logic. Information and Computation 137(1), $1-33$.

Fine, K. (2017). Truthmaker semantics. In C. W. B. Hale and A. Miller (Eds.), A Companion to the Philosophy of Language. John Wiley \& Sons, Ltd, Chichester, UK (online).

Fox, C. and S. Lappin (2008). Foundations of Intensional Semantics. Oxford: Blackwell.

Gallin, D. (1975). Intensional and higher-order modal logic: With applications to Montague semantics, Volume 19. Elsevier.

Groenendijk, J. and M. Stokhof (1991). Dynamic predicate logic. Linguistics and philosophy 14(1), 39-100.

Heim \& von Fintel (2011). Intensional semantics: Mit spring 2011 edition. Retrieved from http://mit.edu/fintel/IntensionalSemantics.pdf.

Kripke, S. A. (1980). Naming and Necessity. Cambride, MA: Harvard University Press.

Leitgeb, H. (2018). HYPE: A system of hyperintensional logic (with an application to semantic paradoxes). Journal of Philosophical Logic, 1-101.

Lewis, D. (1978). Truth in fiction. American philosophical quarterly 15(1), 37-46.

Lewis, D. (1979). Attitudes de dicto and de se. The philosophical review 88(4), 513-543.

MacFarlane, J. (2014). Assessment sensitivity: Relative truth and its applications. OUP Oxford.

Shan, C.-C. (2002). Monads for natural language semantics. In Proceedings of the ESSLLI 2001 Student Session, pp. 285-298.

Shan, C.-C. (2007). Linguistic side effects. In C. Barker and P. Jacobson (Eds.), Direct compositionality, pp. 132-163. Oxford: Oxford studies in theoretical linguistics,.

Veltman, F. (1985). Logic for Conditionals. Ph. D. thesis, University of Amsterdam.

Zimmermann, T. E. (2005). What's in two names? Journal of Semantics 22(1), 53-96.

Zimmermann, T. E. (2012). Compositionality problems and how to solve them. Oxford handbook of compositionality, 81-106. 